\title{
Research Article Existence and Approximation of Fixed Points for Set-Valued Mappings
}

\author{
Simeon Reich and Alexander J. Zaslavski \\ Department of Mathematics, The Technion-Israel Institute of Technology, 32000 Haifa, Israel \\ Correspondence should be addressed to Simeon Reich, sreich@techunix.technion.ac.il \\ Received 8 December 2009; Accepted 29 January 2010 \\ Academic Editor: Tomonari Suzuki
}

Copyright (C) 2010 S. Reich and A. J. Zaslavski. This is an open access article distributed under the Creative Commons Attribution License, which permits unrestricted use, distribution, and reproduction in any medium, provided the original work is properly cited.

Taking into account possibly inexact data, we study both existence and approximation of fixed points for certain set-valued mappings of contractive type. More precisely, we study the existence of convergent iterations in the presence of computational errors for two classes of set-valued mappings. The first class comprises certain mappings of contractive type, while the second one contains mappings satisfying a Caristi-type condition.

\section{Introduction}

The study of the convergence of iterations of mappings of contractive type has been an important topic in Nonlinear Functional Analysis since Banach's seminal paper [1] on the existence of a unique fixed point for a strict contraction [2-5]. Banach's celebrated theorem also yields convergence of iterates to the unique fixed point. During the last fifty years or so, many developments have taken place in this area. Interesting results have also been obtained regarding set-valued mappings, where the situation is more difficult and less understood. See, for example, [5-12] and the references cited therein. As already mentioned above, one of the methods used for proving the classical Banach theorem is to show the convergence of Picard iterations, which holds for any initial point. In the case of set-valued mappings, we do not have convergence of all trajectories of the dynamical system induced by the given mapping. Convergent trajectories have to be constructed in a special way. For instance, in [7], if at the moment $t=0,1, \ldots$ we have reached a point $x_{t}$, then we choose an element of $T\left(x_{t}\right)$ (here $T$ is the given mapping) such that $x_{t+1}$ approximates the best approximation of $x_{t}$ from $T\left(x_{t}\right)$. Since our mapping acts on a general complete metric space, we cannot, in general, choose $x_{t+1}$ as the best approximation of $x_{t}$ by elements of $T\left(x_{t}\right)$. Instead, we require $x_{t+1}$ to approximate the best approximation up to a positive number $\epsilon_{t}$, such that the sequence $\left\{\epsilon_{t}\right\}_{t=0}^{\infty}$ is summable. This method allowed Nadler [7] to obtain the existence of a fixed point of a strictly contractive set-valued mapping and the authors of [6] to obtain more general 
results. In view of this state of affairs, it is important to study convergence of the iterates of set-valued mappings in the presence of errors.

In this paper, we study the existence of convergent iterations in the presence of computational errors for two classes of set-valued mappings. The first class comprises certain mappings of contractive type, while the second one contains mappings satisfying a Caristitype condition.

As we have already mentioned, the existence of a convergent iterative sequence for setvalued strict contractions was established by Nadler [7]. For a more general class of mappings satisfying a certain contractive condition, this was proved in [11]. In the present paper, we show that the existence result of [11] still holds even when possible computational errors are taken into account (see Theorems 2.2-2.4 below).

In Section 3, we obtain certain results regarding set-valued mappings satisfying a Caristi-type condition which complement the results in [6]. There we establish the existence of a fixed point for such mappings assuming that their graphs are closed. Here we first show that a set-valued mapping satisfies a Caristi-type condition if and only if there exists an iterative sequence $\left\{x_{i}\right\}_{i=1}^{\infty}$ such that the sum of the distances between $x_{i}$ and $x_{i+1}$, when $i$ runs from zero to infinity, is finite. Then we prove an analog of the Caristi-type result in [6], replacing the closedness of the graph of the mapping with a lower semicontinuity assumption as in Caristi's original theorem [13].

\section{Set-Valued Mappings of Contractive Type}

Let $(X, \rho)$ be a complete metric space. For each $x \in X$ and each nonempty set $A \subset X$, set

$$
\rho(x, A)=\inf \{\rho(x, y): y \in A\} \text {. }
$$

For each pair of nonempty sets $A, B \subset X$, put

$$
H(A, B)=\max \left\{\sup _{x \in A} \rho(x, B), \sup _{x \in B} \rho(x, A)\right\} .
$$

Let $T: X \rightarrow 2^{X} \backslash\{\emptyset\}, x_{*} \in X$ satisfy

$$
x_{*} \in T x_{*}
$$

let $\phi:[0, \infty) \rightarrow[0,1)$ be a decreasing function such that

$$
\phi(t)<1 \quad \forall t \in[0, \infty),
$$

and assume that

$$
H\left(T(x), T\left(x_{*}\right)\right) \leq \phi\left(\rho\left(x, x_{*}\right)\right) \rho\left(x, x_{*}\right) \quad \forall x \in X
$$

We begin with the following obvious fact. 
Lemma 2.1. Let $x_{0} \in X, \delta>0$, and let a sequence of mappings $T_{i}: X \rightarrow 2^{X} \backslash\{\emptyset\}, i=0,1, \ldots$, satisfy

$$
H\left(T_{i}(x), T(x)\right) \leq \delta, \quad i=0,1, \ldots, x \in X
$$

Then there exist sequences $\left\{x_{i}\right\}_{i=0}^{\infty} \subset X$ and $\left\{y_{i}\right\}_{i=1}^{\infty} \subset X$ such that for any integer $i \geq 0$,

$$
\begin{gathered}
y_{i+1} \in T\left(x_{i}\right), \\
\rho\left(x_{*}, y_{i+1}\right) \leq \rho\left(x_{*}, T x_{i}\right)+\delta, \\
x_{i+1} \in T_{i}\left(x_{i}\right), \\
\rho\left(x_{i+1}, y_{i+1}\right) \leq \rho\left(y_{i+1}, T_{i} x_{i}\right)+\delta .
\end{gathered}
$$

Theorem 2.2. Let $\epsilon$ and $M$ be positive. Then there exist $\delta \in(0, \epsilon)$ and a natural number $n_{0}$ such that for each sequence of mappings $T_{i}: X \rightarrow 2^{X} \backslash\{\emptyset\}, i=0,1, \ldots$, satisfying

$$
H\left(T_{i}(x), T(x)\right) \leq \delta, \quad i=0,1, \ldots, x \in X,
$$

and each $x \in X$ satisfying

$$
\rho\left(x, x_{*}\right) \leq M,
$$

there is a sequence $\left\{x_{i}\right\}_{i=0}^{\infty} \subset X$ such that

$$
x_{0}=x, \quad x_{i+1} \in T_{i}\left(x_{i}\right), \quad i=0,1, \ldots,
$$

and the inequality

$$
\rho\left(x_{i}, x_{*}\right) \leq \epsilon
$$

holds for all integers $i \geq n_{0}$.

This theorem is a consequence of Lemma 2.1 and our next result.

Theorem 2.3. Let $\epsilon \in(0,1)$. Then there exists $\delta \in(0, \epsilon)$ so that for each $M>0$, there is a natural number $n_{0}$ such that the following assertion holds.

Assume that a sequence of mappings $T_{i}: X \rightarrow 2^{X} \backslash\{\emptyset\}, i=0,1, \ldots$, satisfies

$$
H\left(T_{i}(x), T(x)\right) \leq \delta, \quad i=0,1, \ldots,
$$

for all $x \in X$, a sequence $\left\{x_{i}\right\}_{i=0}^{\infty} \subset X$ satisfies

$$
\rho\left(x_{0}, x_{*}\right) \leq M, \quad x_{i+1} \in T_{i}\left(x_{i}\right), \quad i=0,1, \ldots,
$$


and that for each integer $i \geq 0$, there is $y_{i+1} \in T\left(x_{i}\right)$ such that

$$
\begin{gathered}
\rho\left(x_{*}, y_{i+1}\right) \leq \rho\left(x_{*}, T x_{i}\right)+\delta \\
\rho\left(x_{i+1}, y_{i+1}\right) \leq \rho\left(y_{i+1}, T_{i} x_{i}\right)+\delta .
\end{gathered}
$$

Then $\rho\left(x_{i}, x_{*}\right) \leq \epsilon$ for all integers $i \geq n_{0}$.

Proof. Choose a positive number $\delta<\epsilon / 12$ such that

$$
6 \delta \leq\left(\frac{\epsilon}{2}\right)\left(1-\phi\left(\frac{\epsilon}{2}\right)\right)
$$

Let $M>0$. Fix a natural number $n_{0}$ such that

$$
n_{0}>4+\frac{M}{\delta}
$$

Assume that a sequence of mappings $T_{i}: X \rightarrow 2^{X} \backslash\{\emptyset\}, i=0,1, \ldots$, satisfies $(2.12),\left\{x_{i}\right\}_{i=0}^{\infty} \subset X$ satisfies (2.13), and that for each integer $i \geq 0$, there is

$$
y_{i+1} \in T\left(x_{i}\right)
$$

such that (2.14) and (2.15) hold.

Let $i \geq 0$ be an integer. By (2.3), (2.5), and (2.14),

$$
\begin{aligned}
\rho\left(x_{*}, y_{i+1}\right) & \leq \rho\left(x_{*}, T\left(x_{i}\right)\right)+\delta \leq H\left(T\left(x_{*}\right), T\left(x_{i}\right)\right)+\delta \\
& \leq \phi\left(\rho\left(x_{i}, x_{*}\right)\right) \rho\left(x_{i}, x_{*}\right)+\delta .
\end{aligned}
$$

By (2.15), (2.18), and (2.12),

$$
\rho\left(x_{i+1}, y_{i+1}\right) \leq \rho\left(y_{i+1}, T_{i}\left(x_{i}\right)\right)+\delta \leq H\left(T\left(x_{i}\right), T_{i}\left(x_{i}\right)\right)+\delta \leq 2 \delta .
$$

By (2.19) and (2.20),

$$
\rho\left(x_{*} \cdot x_{i+1}\right) \leq \rho\left(x_{*}, y_{i+1}\right)+\rho\left(y_{i+1}, x_{i+1}\right) \leq \phi\left(\rho\left(x_{i}, x_{*}\right)\right) \rho\left(x_{i}, x_{*}\right)+3 \delta
$$

for all integers $i \geq 0$.

We claim that there is an integer $j \in\left\{0, \ldots, n_{0}\right\}$ such that

$$
\rho\left(x_{j}, x_{*}\right) \leq \frac{\epsilon}{2} .
$$


Fixed Point Theory and Applications

Assume the contrary. Then

$$
\rho\left(x_{j}, x_{*}\right)>\frac{\epsilon}{2}, \quad j=0, \ldots, n_{0}
$$

By (2.21), (2.23), and (2.16), we have, for all integers $j \in\left\{0, \ldots, n_{0}-1\right\}$,

$$
\begin{aligned}
\rho\left(x_{*}, x_{j}\right)-\rho\left(x_{*}, x_{j+1}\right) & \geq \rho\left(x_{*}, x_{j}\right)-\phi\left(\rho\left(x_{j}, x_{*}\right)\right) \rho\left(x_{j}, x_{*}\right)-3 \delta \\
& \geq \rho\left(x_{*}, x_{j}\right)\left(1-\phi\left(\rho\left(x_{j}, x_{*}\right)\right)\right)-3 \delta \\
& \geq\left(\frac{\epsilon}{2}\right)\left(1-\phi\left(\frac{\epsilon}{2}\right)\right)-3 \delta \geq \delta .
\end{aligned}
$$

When combined with (2.13), this implies that

$$
M \geq \rho\left(x_{*}, x_{0}\right) \geq \rho\left(x_{*}, x_{0}\right)-\rho\left(x_{*}, x_{n_{0}}\right)=\sum_{j=0}^{n_{0}-1}\left[\rho\left(x_{*}, x_{j}\right)-\rho\left(x_{*}, x_{j+1}\right)\right] \geq n_{0} \delta .
$$

This, however, contradicts (2.17).

Therefore, there is an integer $j \in\left\{0, \ldots, n_{0}\right\}$ such that (2.22) holds. Next, we assert that

$$
\rho\left(x_{i}, x_{*}\right) \leq \epsilon \quad \forall \text { integers } i \geq j .
$$

Assume the contrary. Then there is an integer $p>j$ such that

$$
\rho\left(x_{p}, x_{*}\right)>\epsilon, \quad \rho\left(x_{i}, x_{*}\right) \leq \epsilon \quad \forall \text { integers } i \text { satisfying } j \leq i<p .
$$

There are two cases: either

$$
\rho\left(x_{p-1}, x_{*}\right) \leq \frac{\epsilon}{2}
$$

or

$$
\rho\left(x_{p-1}, x_{*}\right)>\frac{\epsilon}{2}
$$

Assume first that (2.28) holds. By (2.21), (2.28), and (2.16),

$$
\rho\left(x_{p}, x_{*}\right) \leq \rho\left(x_{p-1}, x_{*}\right)+3 \delta \leq \frac{\epsilon}{2}+3 \delta \leq \epsilon .
$$


Now assume that (2.29) holds. By (2.29), (2.21), (2.27), and (2.16),

$$
\begin{aligned}
\rho\left(x_{p}, x_{*}\right) & \leq \phi\left(\rho\left(x_{p-1}, x_{*}\right)\right) \rho\left(x_{p-1}, x_{*}\right)+3 \delta \\
& \leq \phi\left(\frac{\epsilon}{2}\right) \epsilon+3 \delta \leq \phi\left(\frac{\epsilon}{2}\right) \epsilon+\epsilon\left(1-\phi\left(\frac{\epsilon}{2}\right)\right)=\epsilon .
\end{aligned}
$$

This contradicts (2.27).

The contradiction we have reached in both cases proves that (2.26) holds.

This completes the proof of Theorem 2.3.

We end this section with another consequence of Theorem 2.3.

Theorem 2.4. Let $\left\{\epsilon_{i}\right\}_{i=0}^{\infty} \subset(0, \infty), T_{i}: X \rightarrow 2^{X} \backslash\{\emptyset\}, i=0,1, \ldots$, and assume that, for all $x \in X$,

$$
H\left(T x, T_{i}(x)\right) \leq \epsilon_{i}, \quad i=1,2, \ldots, \quad \lim _{i \rightarrow \infty} \epsilon_{i}=0
$$

Then for each $x \in X$, there is a sequence $\left\{x_{i}\right\}_{i=0}^{\infty} \subset X$ such that

$$
x_{0}=x, \quad x_{i+1} \in T_{i} x_{i}, \quad i=0,1, \ldots, \quad \lim _{i \rightarrow \infty} x_{i}=x_{*}
$$

Proof. Let $x \in X$. Put $x_{0}=x$ and define a sequence $\left\{x_{i}\right\}_{i=0}^{\infty} \subset X$ by induction so that for each integer $i \geq 0$, there is $y_{i+1} \in T\left(x_{i}\right)$ satisfying

$$
\begin{gathered}
\rho\left(x_{*}, y_{i+1}\right) \leq \rho\left(x_{*}, T x_{i}\right)+\epsilon_{i}, \\
x_{i+1} \in T_{i} x_{i}, \quad \rho\left(y_{i+1}, x_{i+1}\right) \leq \rho\left(y_{i+1}, T_{i} x_{i}\right)+\epsilon_{i} .
\end{gathered}
$$

In order to show that

$$
\lim _{i \rightarrow \infty} \rho\left(x_{i}, x_{*}\right)=0
$$

we let $\epsilon \in(0,1)$ and prove that for all sufficiently large natural numbers $i$,

$$
\rho\left(x_{i}, x_{*}\right) \leq \epsilon .
$$

Let $\delta \in(0, \epsilon)$ be as guaranteed by Theorem 2.3.

There is a natural number $n_{1}$ such that

$$
\epsilon_{i}<\delta \quad \forall \text { integers } i \geq n_{1} .
$$

Choose $M>0$ such that

$$
\rho\left(x_{n_{1}}, x_{*}\right)<M
$$


Fixed Point Theory and Applications

Let a natural number $n_{0}$ be as guaranteed by Theorem 2.3.

Then for all integers $i \geq n_{0}+n_{1}$,

$$
\rho\left(x_{i}, x_{*}\right) \leq \epsilon
$$

Theorem 2.4 is proved.

\section{Caristi-Type Theorems for Set-Valued Mappings}

We begin this section by recalling [6, Theorem 5.3].

Theorem 3.1. Assume that $(X, \rho)$ is a complete metric space, $T: X \rightarrow 2^{X} \backslash\{\emptyset\}, \operatorname{graph}(T)=$ $\{(x, y) \in X \times X: y \in T x\}$ is closed, $\phi: X \rightarrow R^{1} \cup\{\infty\}$ is bounded from below, and that for each $x \in X$,

$$
\inf \{\phi(y)+\rho(x, y): y \in T x\} \leq \phi(x)
$$

Let $\left\{\epsilon_{n}\right\}_{n=0}^{\infty} \subset(0, \infty), \sum_{n=0}^{\infty} \epsilon_{n}<\infty$, and let $x_{0} \in X$ satisfy $\phi\left(x_{0}\right)<\infty$. Assume that for each integer $n \geq 0, x_{n+1} \in T\left(x_{n}\right)$ and

$$
\phi\left(x_{n+1}\right)+\rho\left(x_{n}, x_{n+1}\right) \leq \inf \left\{\phi(y)+\rho(x, y): y \in T\left(x_{n}\right)\right\}+\epsilon_{n} .
$$

Then $\left\{x_{n}\right\}_{n=0}^{\infty}$ converges to a fixed point of $T$.

In the proof of this theorem, we actually showed that $\sum_{i=0}^{\infty} \rho\left(x_{i}, x_{i+1}\right)<\infty$.

It turns out that the existence of such a sequence is actually equivalent to the existence of a function $\phi: X \rightarrow R^{1} \cup\{\infty\}$ which is bounded from below and such that for each $x \in X$,

$$
\inf \{\phi(y)+\rho(x, y): y \in T x\} \leq \phi(x)
$$

More precisely, we are going to prove the following result.

Theorem 3.2. Let $(X, \rho)$ be a complete metric space and $T: X \rightarrow 2^{X} \backslash\{\emptyset\}$. The following conditions are equivalent.

(A) There exists a function $\phi: X \rightarrow R^{1} \cup\{\infty\}$, which is bounded from below and not identically $\infty$, such that for each $x \in X$, inequality (3.3) holds.

(B) There exists a sequence $\left\{x_{n}\right\}_{n=0}^{\infty} \subset X$ such that $x_{n+1} \in T\left(x_{n}\right)$ for all integers $n$ and $\sum_{n=0}^{\infty} \rho\left(x_{n}, x_{n+1}\right)<\infty$.

Proof. The fact that (A) implies (B) was proved in [6]. To show that (B) implies (A), we define, for each $x \in X$,

$$
\phi(x)=\inf \left\{\sum_{i=0}^{\infty} \rho\left(x_{i}, x_{i+1}\right):\left\{x_{i}\right\}_{i=0}^{\infty} \subset X, x_{0}=x, x_{i+1} \in T\left(x_{i}\right) \quad \forall \text { integers } i \geq 0\right\}
$$


Let $x \in X$. Note that $\phi(x)<\infty$ if and only if there is a sequence $\left\{x_{i}\right\}_{i=0}^{\infty} \subset X$ such that $x_{0}=x$, $x_{i+1} \in T\left(x_{i}\right), i=0,1, \ldots$, and

$$
\sum_{i=0}^{\infty} \rho\left(x_{i}, x_{i+1}\right)<\infty
$$

It is sufficient to show that (3.3) holds for all $x \in X$. To this end, let $x \in X$. We may assume that $\phi(x)<\infty$. Let $\epsilon>0$. There is $\left\{x_{i}\right\}_{i=0}^{\infty} \subset X$ such that $x_{0}=x$,

$$
\begin{aligned}
& x_{t+1} \in T x_{i}, \quad i=0,1, \ldots, \\
& \sum_{i=0}^{\infty} \rho\left(x_{i}, x_{i+1}\right) \leq \phi(x)+\epsilon .
\end{aligned}
$$

Then

$$
x_{1} \in T(x), \quad \rho\left(x, x_{1}\right)+\phi\left(x_{1}\right) \leq \rho\left(x, x_{1}\right)+\sum_{i=1}^{\infty} \rho\left(x_{i}, x_{i+1}\right) \leq \phi(x)+\epsilon,
$$

and so,

$$
\inf \{\phi(y)+\rho(x, y): y \in T(x)\} \leq \phi(x)+\epsilon
$$

Since $\epsilon$ is any positive number, we conclude that (3.3) holds. This completes the proof of Theorem 3.2.

It should be mentioned that in Theorem 3.1 we pose an assumption on $T$ without assuming that $\phi$ possesses lower semicontinuity properties, while in the original Caristi theorem no assumption was made on the mapping, but the function $\phi$ was assumed to be lower semicontinuous. In the following result, we obtain a simple analog of Theorem 3.1 for this situation.

Theorem 3.3. Assume that $(X, \rho)$ is a complete metric space, $T: X \rightarrow 2^{X} \backslash\{\emptyset\}$, Tx is closed for each $x \in X, \phi: X \rightarrow R^{1} \cup\{\infty\}$ is a lower semicontinuous function which is bounded from below and not identically $\infty$, and that for each $x \in X$, inequality (3.3) holds. Then $T$ has a fixed point.

Proof. Choose $x_{0} \in X$ such that

$$
\phi\left(x_{0}\right) \leq \inf \{\phi(z): z \in X\}+\frac{1}{8}
$$

By Ekeland's variational principle [14], there is $x_{1} \in X$ such that

$$
\begin{gathered}
\rho\left(x_{0}, x_{1}\right) \leq 1 \\
\phi\left(x_{1}\right)<\phi(z)+\left(\frac{1}{8}\right) \rho\left(z, x_{1}\right) \quad \forall z \in X \backslash\left\{x_{1}\right\} .
\end{gathered}
$$


Let $\epsilon>0$. By (3.3) and (3.10), there exists $z_{\epsilon} \in T x_{1}$ such that

$$
\begin{gathered}
\phi\left(z_{\epsilon}\right)+\rho\left(x_{1}, z_{\epsilon}\right) \leq \phi\left(x_{1}\right)+\epsilon \leq \epsilon+\phi\left(z_{\epsilon}\right)+\left(\frac{1}{8}\right) \rho\left(z_{\epsilon}, x_{1}\right), \\
\left(\frac{1}{2}\right) \rho\left(x_{1}, z_{\epsilon}\right) \leq \epsilon \\
\inf \left\{\rho\left(x_{1}, z\right): z \in T\left(x_{1}\right)\right\} \leq 2 \epsilon .
\end{gathered}
$$

Since $\epsilon$ is any positive number, it follows that $x_{1} \in T\left(x_{1}\right)$. Theorem 3.3 is proved.

\section{Note added in proof}

After our paper was accepted for publication, Pavel Semenov has kindly informed us that our Theorem 3.3 is almost identical with Corollary 1.7 on page 521 of Volume I of the Handbook of Multivalued Analysis by S. Hu and N. S. Papageorgiou, Kluwer Academic Publishers, Dordrecht, 1997. It may be of interest to note that the above authors deduce their Corollary from a set-valued version of Caristi's fixed point theorem, while we use Ekeland's variational principle (which is known to be equivalent to Caristi's fixed point theorem).

\section{Acknowledgments}

This research was supported by the Israel Science Foundation (Grant no. 647/07), the Fund for the Promotion of Research at the Technion, and by the Technion President's Research Fund.

\section{References}

[1] S. Banach, "Sur les opérations dans les ensembles abstraits et leur application aux équations intégrales," Fundamenta Mathematicae, vol. 3, pp. 133-181, 1922.

[2] K. Goebel and W. A. Kirk, Topics in Metric Fixed Point Theory, vol. 28 of Cambridge Studies in Advanced Mathematics, Cambridge University Press, Cambridge, UK, 1990.

[3] K. Goebel and S. Reich, Uniform Convexity, Hyperbolic Geometry, and Nonexpansive Mappings, vol. 83 of Monographs and Textbooks in Pure and Applied Mathematics, Marcel Dekker, New York, NY, USA, 1984.

[4] W. A. Kirk, "Contraction mappings and extensions," in Handbook of Metric Fixed Point Theory, pp. 1-34, Kluwer Academic Publishers, Dordrecht, The Netherlands, 2001.

[5] W. Takahashi, Nonlinear Functional Analysis: Fixed Point Theory and Its Application, Yokohama Publishers, Yokohama, Japan, 2000.

[6] F. S. de Blasi, J. Myjak, S. Reich, and A. J. Zaslavski, "Generic existence and approximation of fixed points for nonexpansive set-valued maps," Set-Valued and Variational Analysis, vol. 17, no. 1, pp. 97 $112,2009$.

[7] S. B. Nadler Jr., "Multi-valued contraction mappings," Pacific Journal of Mathematics, vol. 30, pp. 475$488,1969$.

[8] S. Reich and A. J. Zaslavski, "Convergence of iterates of nonexpansive set-valued mappings," in Set Valued Mappings with Applications in Nonlinear Analysis, vol. 4 of Mathematical Analysis and Applications, pp. 411-420, Taylor \& Francis, London, UK, 2002.

[9] S. Reich and A. J. Zaslavski, "Generic existence of fixed points for set-valued mappings," Set-Valued Analysis, vol. 10, no. 4, pp. 287-296, 2002.

[10] S. Reich and A. J. Zaslavski, "Two results on fixed points of set-valued nonexpansive mappings," Revue Roumaine de Mathématiques Pures et Appliquées, vol. 51, no. 1, pp. 89-94, 2006. 
[11] S. Reich and A. J. Zaslavski, "Generic aspects of fixed point theory for set-valued mappings," in Advances in Nonlinear Analysis: Theory, Methods and Applications, Cambridge Scientific, Cambridge, UK.

[12] B. Ricceri, “Une propriété topologique de l'ensemble des points fixes d'une contraction multivoque à valeurs convexes," Atti della Accademia Nazionale dei Lincei, vol. 81, no. 3, pp. 283-286, 1987.

[13] J. Caristi, "Fixed point theorems for mappings satisfying inwardness conditions," Transactions of the American Mathematical Society, vol. 215, pp. 241-251, 1976.

[14] I. Ekeland, "On the variational principle," Journal of Mathematical Analysis and Applications, vol. 47, pp. 324-353, 1974. 